\title{
Visual Design Thinking: Understanding the role of knowledge visualization in the design thinking process
}

\author{
Sebastian Kernbach ${ }^{1,3}$, Anja Svetina Nabergoj², 3 \\ ${ }^{1}$ Institute of Media and Communication Management, University of St. Gallen, Switzerland \\ ${ }^{2}$ Faculty of Economics, University of Ljubljana, Slovenia \\ ${ }^{3}$ Hasso Plattner Institute of Design, Stanford University, United States \\ sebastian.kernbach@unisg.ch, anja@dschool.stanford.edu
}

\begin{abstract}
This paper sheds light on the role of visualization methods within the design thinking process. It provides a conceptual framework showing illustrative examples of visualization for each phase indicating its functions and benefits. Based on a thorough understanding of the functions and benefits of knowledge visualization in general, this paper seeks to provide a first overview of the use of visualization in design thinking informing and supporting practitioners and researcher for more conscious selections of visualization methods in their design thinking efforts.
\end{abstract}

Keywords—design thinking, knowledge visualization, visual thinking, visual artifacts, conceptual framework

\section{INTRODUCTION}

Design thinking is the application of designer's tools, processes and mind-sets to non-design domains such as IT, healthcare or business in general to tackle wicked problems [1]. At the forefront of the development of design thinking was the design company IDEO and Stanford Hasso Plattner Institute of Design, also known as d.school, who introduced design thinking to business problems but also to problems of the society at large, e.g. blood donation [2]. In recent years, design thinking has become very popular among organizations and universities around the world.

In design thinking, inter-disciplinary teams work on design challenges in which they aim to identify where the problem is by empathizing with the user through observation and interviewing. Based on this understanding, they aim to define a problem statement that inspires and guides solution finding which is also known as "point of view" (POV). Based on this problem statement, ideas on how to find solutions to that problems are created, firstly by thinking divergently about possible solutions and then choosing converging the best possible solution. Based on these ideas, prototypes are created in the form of tangible physical objects or drawings that invite users and team members to test them, give feedback and refine the solutions [3].

The use of visualization in design thinking is fundamental as it helps to share and communicate ideas, create common understanding, speeds up the process for more and faster innovation cycles, and enables insights leading to action [4]. However, while the use of PowerPoint and bullet lists is limited due to constrains $[5,6]$ and 'being visual' is emphasized, the use of visualization methods in design thinking remains largely applied without neither reflection nor academic investigation. Some exceptions are the work on diagrams in design thinking [7] and the role of visual support for group facilitation [8]. Therefore, a better understanding is necessary of the different forms of visuals, their use in specific phases within design thinking, what principles they convey and how they affect the cognitive, emotional and social dimensions of design thinking work.

This paper aims to contribute to the body of research on design thinking by establishing a conceptual framework of knowledge visualization within the design thinking process. In this first modest attempt, we aim to map key visuals into the different stages of the design thinking process and describe their functions and benefits. In addition, we will provide an overview of future research avenues to inform and motivate future empirical research.

\section{RELATED WORK IN KNOWLEDGE VISUALIZATION}

The application and benefits of knowledge visualization have been widely discussed in fields such as strategy [9], knowledge management [10] and psychology [11]. However, the paramount role of visual reasoning in many instances of problem solving has not been recognized yet [12].

In order to fully understand how design thinking teams can benefit from using visualization, we need to understand the role of visualization through different theoretical lenses. The human brain processes visual information more efficiently compared to written information: when the same information is provided both in written and visual form (such as with a key-word and a corresponding icon), performances are enhanced. According to Dual Coding Theory [13], this effect is because our brain processes visual information and verbal/textual information in two different areas of the brain.

Visually displaying ideas through a visualization offers the benefits to enable participants to externalize 
thoughts and their connections [14] using both their verbal and visual channel [15], thus making it easier for the discussants to build on each other's ideas [16] and to remember the discussed topics better [17]. Mapping the dialogue visually can help overcome cognitive constraints, such as information overload [18] and the finite amount of working memory claimed by cognitive load theory and thus visualizations help to unload our working memory to have more capacity for sense making [19]. In addition, visualizations have the following cognitive, emotional and social functions in collaboration, which design thinking teams can benefit from [20]:

- Cognitive functions

o Facilitating elicitation and synthesis

o Enabling new perspectives

o More exhaustive comparisons

o Easier recall

- Emotional functions

o Creating involvement and engagement

o Providing inspiration

o Providing convincing communication

- Social functions
o Integrating different perspectives
o Assisting mutual understanding
o Showing interdependencies

Based on this generic understanding of the value of knowledge visualization, we aim to showcase its functions more specifically within the context of design thinking. Therefore, the next section shows the conceptual framework and one visualization method for each of the five design thinking process steps together with it functions and benefits.

\section{VISUALIZATION METHODS IN DESIGN THINKING}

When and how can knowledge visualization support design thinking activities? Based on the suggested benefits of knowledge visualization in the previous section, we explore more closely at which points of the design thinking process what type of visualization can be most effectively employed.

We start by taking the design thinking process established at Stanford d.school which is characterised by five stages - empathize, define, ideate, prototype and test, and, by synthesising the results of the related work in knowledge visualization presented above, considering how visualization can support the main function at each stage. We must, however, recognize that the steps offered here in a sequential logic for the clarity of the argumentation - are often cyclical in reality [21].

This synthesis aims to address the following questions for each stage of the design thinking process:

- What type of content needs to be represented? (content);

- What are the expected advantages of using visualization for design thinking? (benefits); and

- What are the appropriate visualization formats or methods that can be used? (visualization methods).

TABLE I. CONCEPTUAL FRAMEWORK OF KNOWLEDGE VISUALIZATION IN DESIGN THINKING

\begin{tabular}{|c|c|c|c|c|c|}
\hline $\begin{array}{l}\text { Design thinking } \\
\text { phases }\end{array}$ & Empathize & Define & Ideate & Prototype & Test \\
\hline Main function & $\begin{array}{l}\text { Identify the right } \\
\text { user, uncover their } \\
\text { needs, discover } \\
\text { their emotions }\end{array}$ & $\begin{array}{l}\text { Unpack and } \\
\text { synthesize findings } \\
\text { into needs and } \\
\text { insights, scope a } \\
\text { meaningful and } \\
\text { actionable problem } \\
\text { statement }\end{array}$ & $\begin{array}{l}\text { Idea generation } \\
\text { (diverging), idea } \\
\text { selection } \\
\text { (converging) }\end{array}$ & $\begin{array}{l}\text { Translate ideas into } \\
\text { tangible artefact, } \\
\text { enable user and } \\
\text { team to interact } \\
\text { with prototype }\end{array}$ & $\begin{array}{l}\text { Refine the solution, } \\
\text { enable user and } \\
\text { team to give } \\
\text { feedback, learn } \\
\text { more about the } \\
\text { user }\end{array}$ \\
\hline Content view & $\begin{array}{l}\text { Stakeholder, needs, } \\
\text { emotions, }\end{array}$ & $\begin{array}{l}\text { Findings, needs, } \\
\text { insights }\end{array}$ & Ideas & $\begin{array}{l}\text { Application of } \\
\text { ideas }\end{array}$ & Opinions \\
\hline Benefits view & $\begin{array}{l}\text { Associate lateral } \\
\text { thinking, } \\
\text { configuration, } \\
\text { perspective and } \\
\text { level switches }\end{array}$ & $\begin{array}{l}\text { Aggregation of } \\
\text { data, facilitate } \\
\text { elicitation and } \\
\text { synthesis, insight } \\
\text { enabler }\end{array}$ & $\begin{array}{l}\text { Compilation space, } \\
\text { associate thinking, } \\
\text { enabling new } \\
\text { perspectives, more } \\
\text { exhaustive } \\
\text { comparisons }\end{array}$ & $\begin{array}{l}\text { Creating } \\
\text { involvement and } \\
\text { engagement, } \\
\text { provide inspiration, } \\
\text { tracking and } \\
\text { showing } \\
\text { interdependencies }\end{array}$ & $\begin{array}{l}\text { Filter function, } \\
\text { showing missing } \\
\text { information, } \\
\text { conflict mediator, } \\
\text { documentation }\end{array}$ \\
\hline $\begin{array}{l}\text { Visualization } \\
\text { methods view }\end{array}$ & $\begin{array}{l}\text { Mind map, } \\
\text { stakeholder map, } \\
\text { empathy map, } \\
\text { conceptual map of } \\
\text { gathered data, } \\
\text { laddering interview } \\
\text { maps }\end{array}$ & $\begin{array}{l}\text { Concept maps, } \\
\text { matrix, Venn } \\
\text { diagram, Personas }\end{array}$ & $\begin{array}{l}\text { Diverging: } \\
\text { Collaborative } \\
\text { sketches, nugget } \\
\text { frame, duo mind } \\
\text { map, brain writing } \\
\text { Converging: } \\
\text { Conceptual } \\
\text { diagrams, matrix, } \\
\text { Venn diagram, dot } \\
\text { voting }\end{array}$ & $\begin{array}{l}\text { Low resolution } \\
\text { prototypes: } \\
\text { Sketches, mock- } \\
\text { ups, customer } \\
\text { journeys, Sankey } \\
\text { diagram, } \\
\text { Confluence } \\
\text { diagram } \\
\text { High resolution } \\
\text { prototypes: } \\
\text { physical objects }\end{array}$ & $\begin{array}{l}\text { Plus-delta feedback } \\
\text { grid, feedback } \\
\text { capture grid, PPCO } \\
\text { feedback form }\end{array}$ \\
\hline
\end{tabular}


These three levels or perspectives, when mapped with the stages of design thinking, build our framework for knowledge visualization in design thinking (Table 1). This framework is in line with the suggestions of MacInnis, as we propose a conceptual framework with the goal of explicating and delineating the role of knowledge visualization within the design thinking process [22]. The framework contains various indicators for the practice of design thinking: in the content view, the framework shows the information that should be visualised based on the main function of that particular phase, in the benefit view it emphasises the benefit to be achieved by applying visualization while the visualization methods view indicates which tools and methods should be used.

\section{A. Empathize}

The main function of this phase is for the design thinking team to observe, engage and immerse with the user and their experiences. It is about learning to see things with "a fresh set of eyes". At the core of this phase is to identify the right users, uncovering their needs and discovering their emotions which guides all innovation efforts. In order to identify the rights users, stakeholder maps are produced to understand the different kind of users, how they are connected and within which systems they operate.

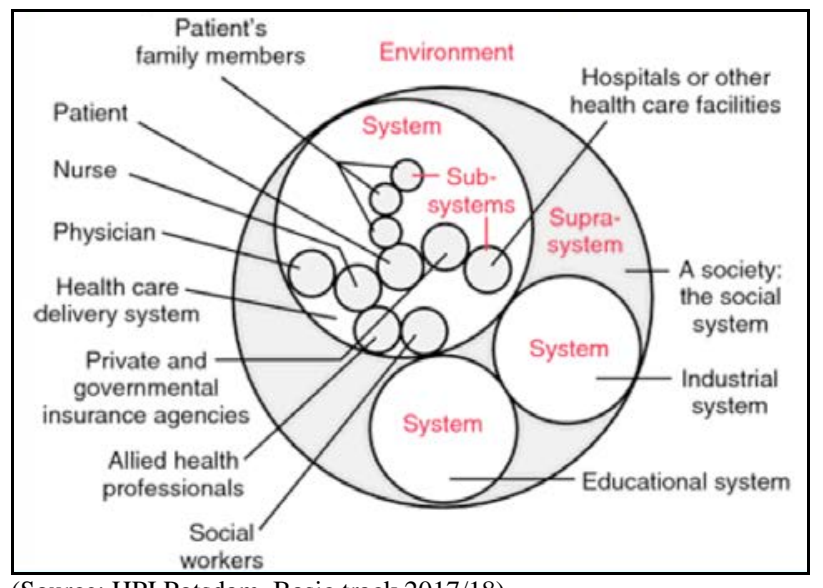

(Source: HPI Potsdam, Basic track 2017/18)

Fig. 1. Stakeholder map

Main function: Identify the rights users and understand their context

What is represented: The stakeholders (e.g. nurse, family members) and the systems (e.g. health care delivery system) that the user (patient) is surrounded by

How it is visualized: with a conceptual diagram using position and size for additional meaning

Medium: computer software (e.g. PowerPoint), whiteboard, paper, or flipchart with post-it-notes

In this phase of divergent thinking, it is important for the design thinking team to visualize the user and other stakeholders, in order to make the context the user is in tangible and to clarify it, but not prematurely commit to any solution design. While verbal and textual information is abstract, visualization makes concepts more concrete and thus manageable. Visualizing the stakeholder and the systems as part of the context is also useful for discussing it with other team members, coaches and even the project partners, who can more easily "see" the key user and other stakeholders and give feedback or contributions. In particular sketching a stakeholder map on paper allows for easy modifications and additions, and this in a collaborative mode; it allows rapid changes and facilitates the emergence of ideas or options.

\section{B. Define}

The main function of this phase is to unpack and synthesize findings into needs and insights to scope a specific and meaningful design challenge. This includes to develop a deep understanding of the user and coming up with an actionable problem statement, also known as "point of view" (POV). The POV is a guiding statement based on specific users, needs and insights and acts as a unique design vision. A good POV frames the problem, captures the heart and mind of the user, inspires the team and acts as a reference for competence ideas.

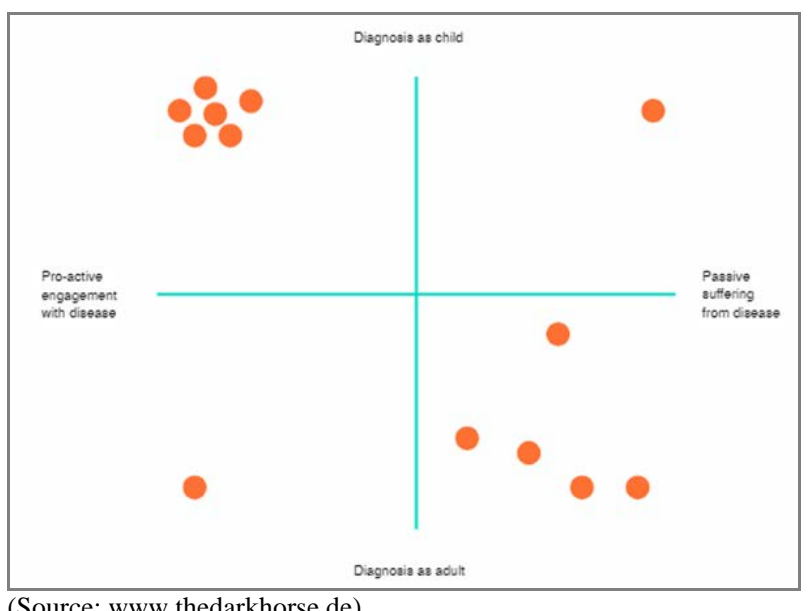

Fig. 2. Age-activity connection for chronically ill people regarding coping activities

Main function: Synthesizing findings into needs and insights to scope the design challenge

What is represented: The interviewed and observed users organized in two dimensions: Time of diagnosis (as child vs. as adult) and type of coping activity (pro-active engagement with the disease vs. passive suffering from disease)

How it is visualized: with a conceptual visualization, using a 2-by-2 matrix as template

Medium: computer software, e.g. PowerPoint

Conceptual templates such as a Matrix and Venn diagram help to synthesize and analyze the findings. The dimensions of the templates typically derive from the data about the user, in the example, the time of diagnosis and type of coping activity of chronically ill people. The visual 
templates are used as a large scale poster with post-its, occasionally also with a software application.

\section{Ideate}

The main function of the "Ideate" phase is idea generation which includes exploring wide solution spaces and focusing on the quantity and diversity of ideas before evaluating and choosing ideas. The distinction of diverging phases (creating options) and converging phases (choosing options) is critical to the quantity, originality and feasibility of ideas. In the convergent phase, evaluation, voting and synthesizing of ideas is critical for the later phases. This phase represents the transition from identifying problems to exploring solutions for users.

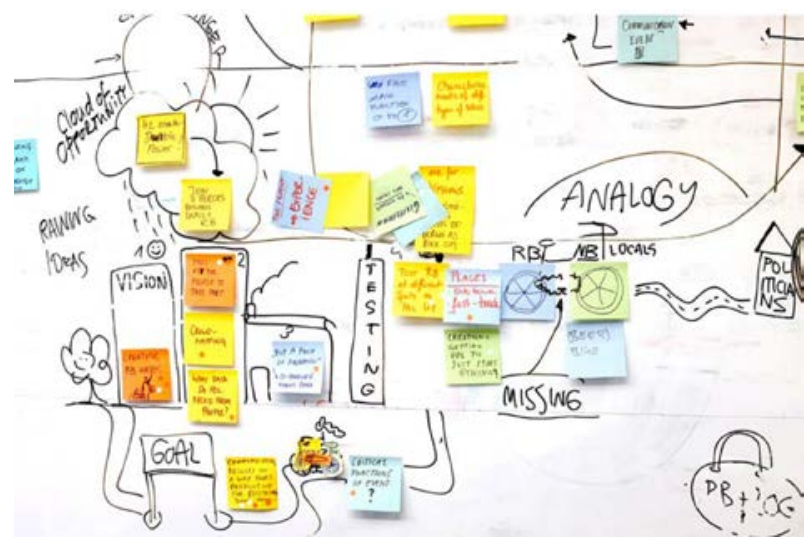

(Source: HPI Potsdam, Basic track 2017/18)

Fig. 3. Free ideation session for a multidimensional urban development project "Radbahn"

Main function: Enabling free visualization of ideas for a solution of the problem

What is represented: The idea spectrum of all team members less how to find solution for creating safer options for biking in the local neighborhood

How it is visualized: with conceptual and metaphoric hand drawings and post-it-notes

Medium: whiteboard, paper, or flipchart

In this phase, design thinkers can rely on simple visualizations such a noting ideas on post-it-notes to impactful visualizations such as visual metaphors. Metaphors offer the advantage that they "convey an insight about the represented information through the key characteristic of the metaphor that is employed" [23]. In Figure 4, the opportunity cloud and the idea of raining ideas is used to communicate the 'opportunities' of the design challenge. The metaphor attracts attention and fosters visual memory in the viewers, compared to text, because it leverages the emotional reactions, and familiarity of (Western) audience with the clouds and rain, suggesting that the opportunity clouds can create rain in the form of further ideas. The range of possible visual metaphors that can be deployed is vast, from iceberg, to roadmaps, and from mountain paths to sailing or racing. In comparison to analytical visualization, such as the Venn diagram in Figure 2, visual metaphors prompt emotional response from the audience and are remembered better.

\section{Prototype}

The main function of the "Prototype" phase is to translate ideas into the physical world and create something tangible. Creating prototypes in early stages should be rough and rapid and are most successful when the user or team can interact with it. Prototypes help to further gain empathy, explore and test ideas and get inspiration in order to learn, solve disagreements, start conversations and fail quickly and cheaply which can be summarized as the solution-building process.

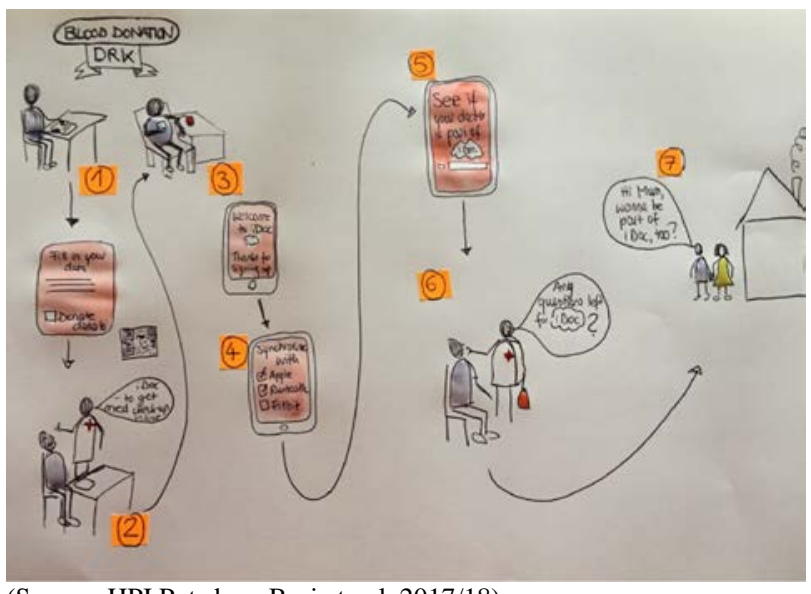

(Source: HPI Potsdam, Basic track 2017/18)

Fig. 4. Customer Journey Mapping of Health Cloud App

Main function: Making the idea of the app explicit and anticipating the customer experience in order to invite feedback from team members and users

What is represented: The customer journey of patients using the Health Cloud App for blood donation

How it is visualized: with conceptual and metaphoric hand drawings

Medium: whiteboard, paper, room/space, or flipchart

Prototypes can be distinguished in low-resolution prototypes such as sketching and drawings to highresolution prototypes such as objects and role play. Visualization is often employed to draw the environment or scene, the props of the experience such as an App and the roles of people associated with the prototype, this result could be a customer journey mapping, storyboards and also concrete sketches of apps and processes. It is important at this stage to produce rough and rapid prototypes that signal "work in progress" [24] and "low perceived finishedness" [25] to invite modification and feedback.

\section{E. Test}

The main function of the "test" phase is to refine the solution by putting the prototypes in the life of the users and 
test the prototypes with them. By testing and refining the prototypes, design thinking teams learn more about the user and might even refine their problem statement aka "point of view" (POV).

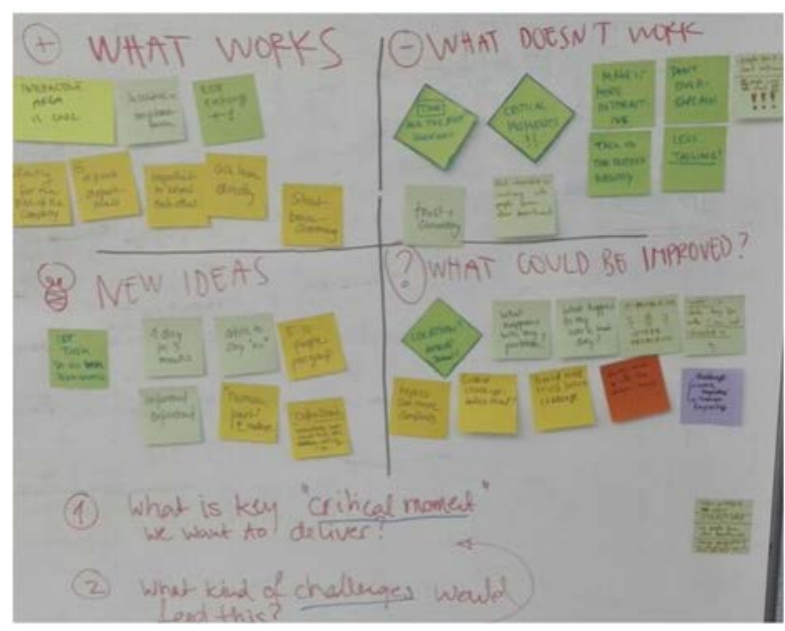

(Source: HPI Potsdam, Basic track 2017/18)

Fig. 5. Feedback grid on the prototype of a team-event on work environment design

Main function: Organizing the feedback into four predefined categories: what works, what does not work, new ideas, and what could be improved

What is represented: Feedback of other team members regarding the prototype of a team-event in which employees of a company could design their own work environment

How it is visualized: with a conceptual hand drawings

Medium: whiteboard, paper, or flipchart with post-itnotes

In opposition to classic innovation approaches, design thinking teams seek to test their prototypes early on with users to learn from their feedback, refine the solutions and build new prototypes. For this reason, prototyping and testing should be done rapidly to fail early and cheaply. Visualization has the power to quickly generate a tangible output by sketching and using post-it's, easily attract attention and trigger emotional response in the audience and make it easy for them to give feedback as the prototypes are signaling “work in progress” [26].

Having described the main functions of each of the five phases of design thinking and given an example of a visualization method to support design thinking teams in their efforts in each phase, the remaining part of this paper covers the limitations and implications and finished with the conclusion.

\section{LIMITATIONS AND IMPLICATIONS}

This paper has been a first attempt to describe visualization methods used in the five phases of design thinking with the aim to inform and support reflection of practitioners and researchers about the role of visualization in design thinking. Limitations arise from the set of examples that were present to the authors, there are many more visualizations in other design thinking projects. We further want to make explicit that those visualization act as illustrative examples and not as the "standard". We also acknowledge that all five phases include divergent and convergent phases and that the visualizations covered in this article cover only one of those two phases.

We hope that by making the functions of visualization explicit in general and showcasing examples for each of the design thinking phases, design thinking practitioners and researchers are inspired to think of and make use of visualizations more consciously in the future. We also hope to create a more sophisticated overview of the current use of visuals within design thinking in the future and add new forms of visuals for design thinking teams, such as in the first attempt of Eppler and Kernbach by introducing the Sankey diagram as well as the Confluence Dynagram to design thinking teams [27].

Future research might investigate the enactment of visualization methods in depths through an ethnographic approach observing and interviewing design thinking practitioners. Quantitative investigation could compare different kind of visualization methods in an experimental setup. In addition, the use of new visualization methods, such as Navicons [28], digital knowledge mapping techniques [29] or Dynagrams [30], could be explored to further enhance the productivity of design thinking teams. One should also not forget the communication between the design thinking team and the (corporate) partner which requires clear and transparent communication of processes and decisions as well as their value. This aspects warrants careful study in the future to better understand the impact of design thinking in the (business) world.

\section{CONCLUSION}

Design thinking is providing a new approach to creativity and innovation that is human-centred, integrating early prototypes and testing with users. It is currently very popular among business innovators and touches even upon all aspects of life including careers, i.e. the Stanford course 'designing your life'.

It provides a new approach including a process, methods and mind-set. A central piece of design thinking in terms of the methods (e.g. be visual) but also in bringing the mindsets alive (e.g. bias-to-action) is the use of visualization. It is therefore not surprising that the first course of design thinking, even long before d.school at Stanford had started, was on visual thinking and its importance for design.

Despite this importance, the use of visualization within design thinking happens with little reflection and knowledge about its functions, benefits and suitability to the situation and goals at hand. This might even hinder or ruin the effectiveness and development of design thinking teams and their solution to the identified problems.

Therefore, this paper is a first attempt to shed light on the use of visual thinking with design thinking by suggesting the conceptual framework and illustrative examples. We 
hope to inspire both practitioners as well as researchers to be more conscious in using visualization methods and better understand how it affects the creativity, effectiveness and efficiency of design thinking (teams).

\section{REFERENCES}

[1] Buchanan, R. (1992). Wicked problems in design thinking. Design issues, 8(2), 5-21.

[2] Cross, N. (2011). Design thinking: Understanding how designers think and work. New York: Bloomsbury Academic.

[3] Brown, T. (2009). Change by design. New York: HarperBusiness.

[4] Shimojima, A. (1999). Derivative meaning in graphical representations. In Visual Languages, Proceedings, IEEE Symposium, 212-219.

[5] Kernbach, S., Bresciani, S., \& Eppler, M. J. (2015). Slipsliding-away: A review of the literature on the constraining qualities of PowerPoint. Business and Professional Communication Quarterly, 78(3), 292-313.

[6] Kernbach, S., Eppler, M. J., \& Bresciani, S. (2015). The use of visualization in the communication of business strategies: An experimental evaluation. International Journal of Business Communication, 52(2), 164-187.

[7] Eppler, M. J., \& Kernbach, S. (2016). Dynagrams: Enhancing design thinking through dynamic diagrams. Design Studies, 47, 91-117.

[8] Eppler, M.J. \& Kernbach, S. (2018). Towards a framework of facilitation in participatory innovation: group collaboration through visual guidance. $5^{\text {th }}$ Participatory Innovation Conference Proceedings (PIN-C), 243-250.

[9] Eppler, M.J., \& Platts, K.W. (2009). Visual Strategizing: The Systematic Use of Visualization in the Strategic-Planning Process. Long Range Planning, 42(1), 42-74.

[10] Eppler, M. J., \& Burkhard, R. A. (2007). Visual representations in knowledge management: framework and cases. Journal of knowledge management, 11(4), 112-122.

[11] Tversky, B. (2005). Visuospatial reasoning. The Cambridge handbook of thinking and reasoning, 209-240.

[12] Goldschmidt, G. (1994). On visual design thinking: the vis kids of architecture. Design studies, 15(2), 158-174.

[13] Clark, J. M., \& Paivio, A. (1991). Dual coding theory and education. Educational psychology review, 3(3), 149-210.

[14] Kernbach, S. (2015). The Facilitative Power of Visual Artifacts for Knowledge Sharing in Client-consultant Interactions. In Academy of Management Proceedings, 14578, Academy of Management.

[15] Paivio, A. (1978). A dual coding approach to perception and cognition. Modes of perceiving and processing information, 39-51.

[16] Mengis, J., \& Eppler, M. J. (2008). Understanding and managing conversations from a knowledge perspective: an analysis of the roles and rules of face-to-face conversations in organizations. Organization Studies, 29(10), 1287-1313.

[17] Mengis, J., \& Eppler, M. J. (2006). Seeing versus arguing the moderating role of collaborative visualization in team knowledge integration. Journal of Universal Knowledge Management, 1(3), 151-162.
[18] O'Reilly, C. A. (1980). Individuals and information overload in organizations: Is more necessarily better? Academy of management journal, 23(4), 684-696.

[19] Simon, H. A. (1972). Theories of bounded rationality. Decision and organization, 1(1), 161-176.

[20] Eppler, M.J., \& Platts, K.W. (2009). Visual Strategizing: The Systematic Use of Visualization in the Strategic-Planning Process. Long Range Planning, 42(1), 42-74.

[21] Brown, T. and Wyatt, J. Design thinking for social innovation. Stanford Social Innovation Review, 8(1), 30-35. 2010.

[22] MacInnis, D. J. (2011). A framework for conceptual contributions in marketing. Journal of Marketing, 75(4), 136154.

[23] Eppler, M. J. The image of insight: The use of visual metaphors in the communication of knowledge. In Proceedings of I-KNOW: Graz, Austria. September 2003.

[24] Suwa, M., \& Tversky, B. (1997). What do architects and students perceive in their design sketches? A protocol analysis. Design studies, 18(4), 385-403.

[25] Bresciani S., Eppler M., and Blackwell A. (2008). A Collaborative Dimensions Framework: Understanding the Mediating Role of Conceptual Visualizations in Collaborative Knowledge Work. In Proceedings of the 41st Hawaii International Conference on System Sciences (HICSS).

[26] Tversky, B. (2002). What do sketches say about thinking. In AAAI Spring Symposium, Sketch Understanding Workshop, Stanford University, AAAI Technical Report SS-02-08, 148151.

[27] Eppler, M. J., \& Kernbach, S. (2016). Dynagrams: Enhancing design thinking through dynamic diagrams. Design Studies, 47, 91-117.

[28] Eppler, M. J., Hoffmann, M. H., \& Kernbach, S. (2015). Navicons for Collaboration-Navigating and Augmenting Discussions through Visual Annotations. In Information Visualisation (iV), IEEE 19th International Conference on Knowledge Visualization and Visual Thinking, 386-391.

[29] Kernbach, S., \& Bresciani, S. (2018). Digital Knowledge Mapping. In North, K., Maier, R. \& Haas, O. (Ed.), Knowledge Management in Digital Change, 129-152. Cham: Springer.

[30] Eppler, M.J. \& Kernbach, S. (2016). Dynagrams: Enhancing Design Thinking through Dynamic Diagrams. In Brenner, W. \& Uebernickel, F. (Ed.). Design thinking for innovation: Research and practice, 85-102. Cham: Springer. 